\title{
Temperature-Induced Changes in Reperfused Stroke: Inflammatory and Thrombolytic Biomarkers
}

\author{
Paulo Ávila-Gómez ${ }^{1}$, Pablo Hervella ${ }^{1}$, Andrés Da Silva-Candal ${ }^{1}$, María Pérez-Mato ${ }^{2}$, \\ Manuel Rodríguez-Yáñez ${ }^{3}$, Iria López-Dequidt ${ }^{3}$, José M. Pumar ${ }^{4}$, José Castillo ${ }^{1}$, \\ Tomás Sobrino $^{1}\left(\mathbb{D}\right.$, Ramón Iglesias-Rey ${ }^{1, *}$ (D) and Francisco Campos ${ }^{1, *}$ (D) \\ 1 Clinical Neurosciences Research Laboratory (LINC), Health Research Institute of Santiago de \\ Compostela (IDIS), E15706 Santiago de Compostela, Spain; paulo.avila.gomez@sergas.es (P.Á.-G.); \\ pablo.hervella.lorenzo@sergas.es (P.H.); andres.alexander.da.silva.candal@sergas.es (A.D.S.-C.); \\ jose.castillo.sanchez@sergas.es (J.C.); tomas.sobrino.moreiras@sergas.es (T.S.) \\ 2 Neuroscience and Cerebrovascular Research Laboratory, Department of Neurology and Stroke Center, \\ La Paz University Hospital, Neuroscience Area of IdiPAZ Health Research Institute, Universidad Autónoma \\ de Madrid, E28046 Madrid, Spain; mery19832005@yahoo.es \\ 3 Stroke Unit, Department of Neurology, Health Research Institute of Santiago de Compostela, Hospital \\ Clínico Universitario, 15706 Santiago de Compostela, Spain; manyanez@yahoo.es (M.R.-Y.); \\ iriaalejandralopez@googlemail.com (I.L.-D.) \\ 4 Department of Neuroradiology, Hospital Clínico Universitario, Health Research Institute of Santiago de \\ Compostela (IDIS), E15706 Santiago de Compostela, Spain; josemanuel.pumar@usc.es \\ * Correspondence: ramon.iglesias.rey@sergas.es (R.I.-R.); francisco.campos.perez@sergas.es (F.C.); \\ Tel./Fax: +34-981951098 (R.I.-R. \& F.C.)
}

Received: 13 June 2020; Accepted: 2 July 2020; Published: 4 July 2020

\begin{abstract}
Although hyperthermia is associated with poor outcomes in ischaemic stroke (IS), some studies indicate that high body temperature may benefit reperfusion therapies. We assessed the association of temperature with effective reperfusion (defined as a reduction of $\geq 8$ points in the National Institute of Health Stroke Scale (NIHSS) within the first $24 \mathrm{~h}$ ) and poor outcome (modified Rankin Scale (mRS) > 2) in 875 retrospectively-included IS patients. We also studied the influence of temperature on thrombolytic (cellular fibronectin ( $\mathrm{FFn}$ ); matrix metalloproteinase 9 (MMP-9)) and inflammatory biomarkers (tumour necrosis factor-alpha (TNF- $\alpha$ ), interleukin 6 (IL-6)) and their relationship with effective reperfusion. Our results showed that a higher temperature at 24 but not $6 \mathrm{~h}$ after stroke was associated with failed reperfusion (OR: 0.373, $p=0.001$ ), poor outcome (OR: 2.190, $p=0.005$ ) and higher IL-6 levels (OR: $0.958, p<0.0001$ ). Temperature at $6 \mathrm{~h}$ was associated with higher MMP-9 levels $(\mathrm{R}=0.697 ; p<0.0001)$ and effective reperfusion, although this last association disappeared after adjusting for confounding factors (OR: 1.178, $p=0.166$ ). Our results suggest that body temperature $>37.5^{\circ} \mathrm{C}$ at $24 \mathrm{~h}$, but not at $6 \mathrm{~h}$ after stroke, is correlated with reperfusion failure, poor clinical outcome, and infarct size. Mild hyperthermia $\left(36.5-37.5^{\circ} \mathrm{C}\right)$ in the first $6 \mathrm{~h}$ window might benefit drug reperfusion therapies by promoting clot lysis.
\end{abstract}

Keywords: biomarkers; ischemic stroke; recanalization therapy; reperfusion; temperature

\section{Introduction}

Temperature is a long-known pivotal factor in the development and progression of neurological injuries, particularly in the field of stroke, where approximately $50 \%$ of patients develop hyperthermia (or fever) within the first $24 \mathrm{~h}[1,2]$. Indeed, higher temperatures during the acute phase of ischaemic stroke (IS) have been associated with greater infarct volumes and worse functional outcomes at 3 months [2-8]. Therapeutic hypothermia helps to preserve tissue energy and halts several cell 
death mechanisms; thus, it is currently considered one of the most promising neuroprotective approaches in preclinical models of stroke $[9,10]$. Nonetheless, although therapeutic hypothermia has well-established neuroprotective effects in specific conditions, its translatability to the human clinic remains elusive [11,12].

Besides mechanical devices, reperfusion drug therapy with recombinant tissue plasminogen activator (rtPA) remains the treatment of choice during the acute phase of an ischaemic event, although its use is limited due to the risk of haemorrhage [13-15]. In this regard, body temperature also has a relevant influence on the efficacy of thrombolytic therapy. Paradoxically, although hyperthermia has been associated with poor outcomes and an increased risk of developing haemorrhagic transformation (HT) after rtPA treatment [2], some studies have shown that high body temperatures could have a beneficial effect on reperfusion therapies by enhancing the thrombolytic activity of rtPA [16-18]. For instance, previous studies have reported increased fibrinolysis using streptokinase at higher temperatures [19], while lower clot lysis was observed at lower temperatures when rtPA was added to clot suspensions [20]. In patients with acute lower limb ischaemia treated with catheter-directed thrombolysis, heating the rtPA also resulted in faster clot lysis [21]. Therefore, it is not clear if the improvement in clot lysis and efficacy of reperfusion can overcome the deleterious effect of hyperthermia on stroke outcome in rtPA-treated patients.

In this regard, it has been widely reported that, although reperfusion is associated with improved patient outcomes, it can also exacerbate temperature-dependent processes related to blood-brain barrier disruption and post-stroke inflammatory response, leading to increased brain damage [22]. For instance, rtPA upregulates matrix metalloproteinase 9 (MMP-9) expression [23], which is associated with HT and poor outcome in IS [24], and hyperthermia exacerbates the destruction of microvascular integrity by increasing MMP-9 activity [25]. Our group has also reported that circulating levels of fibronectin ( $\mathrm{cFn}$ ) predicted rtPA-associated HT after systemic thrombolysis [26]. Moreover, we have previously demonstrated that higher levels of proinflammatory markers such as interleukin 6 (IL-6) and tumour necrosis factor-alpha (TNF- $\alpha$ ) were associated with poor outcomes and larger infarcts in IS patients with hyperthermia [27] and rtPA treatment [28].

Given the conflicting evidence on the effect of temperature on thrombolytic-reperfusion, we hypothesised that higher body temperature during the acute phase of an IS could have a beneficial effect on reperfusion therapies and patient outcomes. Thus, this work aimed to study the influence of temperature on the recanalisation effectiveness and functional outcome at 3 months in a population of IS patients receiving reperfusion therapies. To further support our analysis, we evaluated the effect of temperature on the levels of fibrinolytic and inflammatory activity biomarkers and their impact on the effectiveness of reperfusion. As a secondary objective, we analysed the influence of temperature on the percentage of patients with HT and infarct volume.

\section{Materials and Methods}

\subsection{Study Design}

This retrospective observational study was conducted on a registry of patients with IS included consecutively and prospectively from our databank. The patients were admitted to the Stroke Unit of the University Clinical Hospital of Santiago de Compostela (Spain) from January 2008 to December 2018. The study was performed in accordance with the principles of the Declaration of Helsinki of the World Medical Association and approved by the Research Ethics Committee of Santiago (project identification code 2019/616). Informed consent was obtained from each patient or their relatives after providing a full explanation of the procedures.

\subsection{Inclusion Criteria}

All patients included in this study met the following criteria: (1) authorisation for the anonymous use of their data for research; (2) magnetic resonance imaging (MRI) or computed tomography (CT) 
study at inclusion and between days four and seven; (3) hemispheric location; (4) patients undergoing reperfusion treatment with rtPA alone or in combination with thrombectomy; (5) patients with a latency time between stroke onset and inclusion of $\leq 6 \mathrm{~h}$; and (6) a minimum follow-up (face-to-face or telephone) duration of 3 months. Patients who met any of the following criteria were not considered for the study: (1) institutionalised patients, (2) comorbidity and life expectancy <1 year, (3) without subsequent diagnostic confirmation, (4) lacunar infarctions, and (5) loss to follow-up at 3 months.

\subsection{Blood Samples and Biomarker Assays}

Biochemistry, haematology, and coagulation tests were performed in the central laboratory of the hospital. Measurements were made from blood samples obtained in the first $6 \mathrm{~h}$ after admission and the next $24 \pm 6 \mathrm{~h}$. Although the biomarker analysis was not performed simultaneously, it was supervised by the same researchers and carried out using the same standardised methods. For this study, we selected MMP-9 and cellular cFn as markers associated with thrombolytic activity $[24,26]$, while IL-6 and TNF- $\alpha$ were selected as markers associated with inflammatory activity [27]. For these molecular assessments, venous blood samples were collected in vacutainer tubes (Becton Dickinson, San Jose, CA, USA) in the first $6 \mathrm{~h}$ after symptom onset (always after the administration of the thrombolytic bolus) and at $24 \pm 6 \mathrm{~h}$. After allowing the sample to clot for $60 \mathrm{~min}$, the blood samples were centrifuged at $3000 \times g$ for $10 \mathrm{~min}$ and the serum was immediately aliquoted, frozen, and stored at $-80^{\circ} \mathrm{C}$ until analysis.

Serum levels of IL-6 were measured by enzyme-linked immunosorbent assay (ELISA) (BioLegend, San Diego, CA, USA) with a minimum assay sensitivity of $1.6 \mathrm{pg} / \mathrm{mL}$ and an intra- and inter-assay coefficient of variation (CV) of $5.0 \%$ and $6.8 \%$, respectively, following the manufacturer's instructions. TNF- $\alpha$ was measured using an immunodiagnostic IMMULITE 1000 System (Siemens Healthcare Global, Los Angeles, CA, USA) with a minimum assay sensitivity of $1.7 \mathrm{pg} / \mathrm{mL}$, an intra-assay CV of $3.5 \%$, and an inter-assay CV of 6.5\%. Finally, serum levels of active MMP-9 (GE Healthcare, Amersham, UK, Little Chalfont, Buckinghamshire, UK) and cFn (BioHit, Helsinki, Finland) were measured using commercial ELISA kits following the manufacturer's instructions. The intra- and inter-assay CVs were $<8 \%$. All biomarkers were evaluated within the first 3 months after blood sample collection.

\subsection{Clinical Scale, Temperature and Therapeutic Management}

All patients were admitted to the stroke unit and were treated under the Spanish Neurological Society protocol [29] by trained neurologists experienced in cerebrovascular diseases. The intensity of the neurological deficit was determined by the National Institute of Health Stroke Scale (NIHSS) upon admission to the Stroke Unit. Neurological improvement, defined as a reduction of $\geq 8$ points in the NIHSS in the first $24 \mathrm{~h}$, was used as a clinical marker of effective reperfusion [13]. Functional outcome was assessed at 3 months \pm 15 days (face-to-face in $80.8 \%$ of the sample) using the modified Rankin Scale (mRS) (mRs categorised as poor outcome $>2$ ). Both scales were evaluated by internationally certified neurologists. Temperature was measured by the nursing staff every $6 \mathrm{~h}$. For this analysis, the axillary temperature was measured at the time of admission and at 6 and $24 \mathrm{~h}$. Patients with temperatures $\geq 37.5^{\circ} \mathrm{C}$ were treated with paracetamol (500 mg p.o.) or metamizole ( $2 \mathrm{~g}$ i.v.) every $6 \mathrm{~h}$.

\subsection{Neuroimaging Studies}

Cerebral CT or MRI studies were performed on and between days 4 and 7. All neuroimaging studies were supervised by the same neuroradiologist. Symptomatic HT was assessed at the time of recording the neurological worsening and, in any case, in the follow-up CT. HT was defined as symptomatic when it was associated with early neurological deterioration (worsening of at least 4 points in the NIHSS during the first $48 \mathrm{~h}$ after stroke onset). HT was classified according to the European Cooperative Acute Stroke Study (ECASS) III [30] criteria as follows: haemorrhagic infarction type 1 (HI1) was defined as small petechiae along the infarct margins, HI type 2 (HI2) was defined as more confluent petechiae within the infarct area but without space-occupying effect, parenchymal haemorrhage type 1 (PH1) was defined as blood clots not exceeding $30 \%$ of the infarct with some 
mild space-occupying effect, and PH type 2 (PH2) as blood clots exceeding $30 \%$ of the infarct area with significant space-occupying effect. The $\mathrm{PH} 1$ and $\mathrm{PH} 2$ groups were considered to have severe HT. The initial lesion volume was determined upon admission by diffusion-weighted imaging (DWI) through an automatic planimetric method. Lesion volume was determined in the follow-up CT using the $\mathrm{ABC} / 2$ method [31] until 2016, and then by automated planimetric method.

\subsection{Endpoints}

The main outcome variables were effective reperfusion (defined as a reduction of $\geq 8$ points in the NIHSS in the first $24 \mathrm{~h}$ ) and poor patient outcomes (mRS $>2$ ). Mild hyperthermia was defined as an axillary temperature between $36.5^{\circ} \mathrm{C}$ and $37.5^{\circ} \mathrm{C}$. As secondary endpoints, we also analysed the association between effective reperfusion and serum levels of thrombolytic (MMP-9, cFN) and inflammatory (IL-6, TNF- $\alpha$ ) biomarkers. Finally, we also determined the association between infarct volume and HT with temperature.

\subsection{Statistical Analysis}

In the descriptive analysis, categorical variables were expressed as frequencies and percentage and as means (standard deviation (SD)) or median and interquartile range (25th and 75th percentiles) for the continuous variables, depending on their adjustment to a normal distribution. The normality of the sample was determined by Kolmogorov-Smirnov tests with Lilliefors correction. Statistical inference was then performed with chi-square, Student's t, or Mann-Whitney tests according to the nature of the contrast variable and its adjustment to normality. Bivariate correlations were performed using Pearson's correlations for normally distributed variables.

The association of temperature (at admission and 6 and $24 \mathrm{~h}$ ) with effective reperfusion, outcome at 3 months, HT, and serum levels of the studied biomarkers were assessed using logistic regression analysis models and the correlation between temperature and infarct volume was analysed by linear multivariate regression. Each model was adjusted for the independent variables in the bivariate analysis. The optimal cut-off points were calculated in the variables of interest using receiver operative curve (ROC) analysis. The results were expressed as adjusted odds ratios (ORs) or B with their respective $95 \%$ confidence intervals (CIs). $p$-Values $<0.05$ were considered statistically significant in all tests. All analyses were conducted using IBM SPSS Statistics for Macintosh, version 20.0 (IBM Corp, Armonk, NY, USA).

\section{Results}

\subsection{Sample Description}

The sample is described in Table 1. This study included a total of 875 patients (mean age, $72.0 \pm 12.5$ years; $45.9 \%$ females) with acute IS. The mean temperatures at admission and 6 and 24 were $36.2 \pm 0.6{ }^{\circ} \mathrm{C}, 36.6 \pm 0.7{ }^{\circ} \mathrm{C}$, and $36.5 \pm 0.7^{\circ} \mathrm{C}$, respectively. Regarding the aetiology of the stroke, $43.5 \%$ were of cardioembolic origin, followed by atherothrombotic $(23.5 \%)$ and lacunar $(1.3 \%)$. Undetermined origin or other origins were reported in $31.7 \%$ of cases. Systemic fibrinolysis with rtPA was the reperfusion treatment of choice in $91.1 \%$ of cases, and intravenous or i.a. thrombolysis combined with thrombectomy in the remaining $8.9 \%$ of cases. 
Table 1. Description of the 875 patients included in the study.

\begin{tabular}{|c|c|}
\hline Characteristic & Total Sample $(n=875)$ \\
\hline Age, years & $72.0 \pm 12.5$ \\
\hline Women, \% & 45.9 \\
\hline Onset-treatment time, $\min$ & $161.8 \pm 61.2$ \\
\hline Previous disability (modified Rankin Scale) & $0(0,0)$ \\
\hline Hypertensive, \% & 63.8 \\
\hline Diabetics, $\%$ & 22.9 \\
\hline Smokers, \% & 22.5 \\
\hline Alcohol consumption, $\%$ & 10.3 \\
\hline Dyslipidaemia, $\%$ & 39.3 \\
\hline Peripheral arterial disease, $\%$ & 6.7 \\
\hline Atrial fibrillation, $\%$ & 22.9 \\
\hline Ischemic heart disease, $\%$ & 12.7 \\
\hline Temperature at admission, ${ }^{\circ} \mathrm{C}$ & $36.2 \pm 0.6$ \\
\hline Temperature at $6 \mathrm{~h},{ }^{\circ} \mathrm{C}$ & $36.6 \pm 0.7$ \\
\hline Temperature at $24 \mathrm{~h},{ }^{\circ} \mathrm{C}$ & $36.5 \pm 0.7$ \\
\hline Blood glucose, mg/dL & $138.6 \pm 55.7$ \\
\hline Glycosylated haemoglobin, \% & $6.2 \pm 4.5$ \\
\hline Leukocytes, $\times 10^{3} / \mathrm{mL}$ & $8.3 \pm 3.2$ \\
\hline Fibrinogen, $\mathrm{mg} / \mathrm{dL}$ & $414.2 \pm 103.2$ \\
\hline Microalbuminuria, $\mathrm{mg} / 24 \mathrm{~h}$ & $5.7 \pm 7.8$ \\
\hline C-reactive protein, $\mathrm{mg} / \mathrm{L}$ & $5.5 \pm 4.7$ \\
\hline LDL-cholesterol, mg/dL & $108.0 \pm 40.7$ \\
\hline HDL-cholesterol, mg/dL & $41.7 \pm 14.9$ \\
\hline Triglycerides, mg/dL & $114.2 \pm 51.5$ \\
\hline Sedimentation rate, $\mathrm{mm}$ & $18.3 \pm 20.4$ \\
\hline NIHSS at admission & $17(12,22)$ \\
\hline DWI volume at admission, $\mathrm{mL}$ & $28.1 \pm 43.5$ \\
\hline \multicolumn{2}{|l|}{ Leukoaraiosis } \\
\hline No, $\%$ & 41.7 \\
\hline Fazekas I, \% & 36.5 \\
\hline Fazekas II, \% & 15.0 \\
\hline Fazekas III, \% & 6.9 \\
\hline \multicolumn{2}{|l|}{ Trial of ORG 10172 in acute stroke treatment (TOAST) } \\
\hline Atherothrombotic, \% & 23.5 \\
\hline Cardioembolic, \% & 43.5 \\
\hline Lacunar, \% & 1.3 \\
\hline Undetermined/others, $\%$ & 31.7 \\
\hline \multicolumn{2}{|l|}{ Type of reperfusion treatment } \\
\hline Systemic fibrinolysis, $\%$ & 91.1 \\
\hline IV or IA thrombolysis + thrombectomy, \% & 8.9 \\
\hline Effective reperfusion, $\%$ & 44.0 \\
\hline Early neurological deterioration, $\%$ & 9.9 \\
\hline \multicolumn{2}{|l|}{ Haemorrhagic transformation } \\
\hline No, $\%$ & 68.0 \\
\hline $\mathrm{IH} 1, \%$ & 21.4 \\
\hline $\mathrm{IH} 2, \%$ & 5.3 \\
\hline $\mathrm{PH} 1, \%$ & 3.0 \\
\hline $\mathrm{PH} 2, \%$ & 2.4 \\
\hline Lesion volume at days $4-7, \mathrm{~mL}$ & $51.1 \pm 75.8$ \\
\hline Modified Rankin Scale at 3 months & $1(0,3)$ \\
\hline Good functional outcome at 3 months, $\%$ & 69.3 \\
\hline
\end{tabular}

LDL: low density lipoprotein; HDL: high density lipoprotein; NIHSS: National Institute of Health Stroke Scale; DWI: diffusion-weighted imaging; IH1: infarction type 1; IH2: infarction type 2; PH1: parenchymal haemorrhage type 1; PH2: parenchymal haemorrhage type 1; IV: intravenous; IA: intra arterial. 


\subsection{Analysis of the Association between Temperature and Effective Reperfusion}

Among the studied population, $44 \%$ of the patients had effective reperfusion, while the remaining $56 \%$ did not (385 vs. 490 patients). The patients with effective reperfusion showed lower temperatures both at admission and at $24 \mathrm{~h}$. Interestingly, a higher temperature at $6 \mathrm{~h}$ was associated with successful reperfusion (Figure 1).

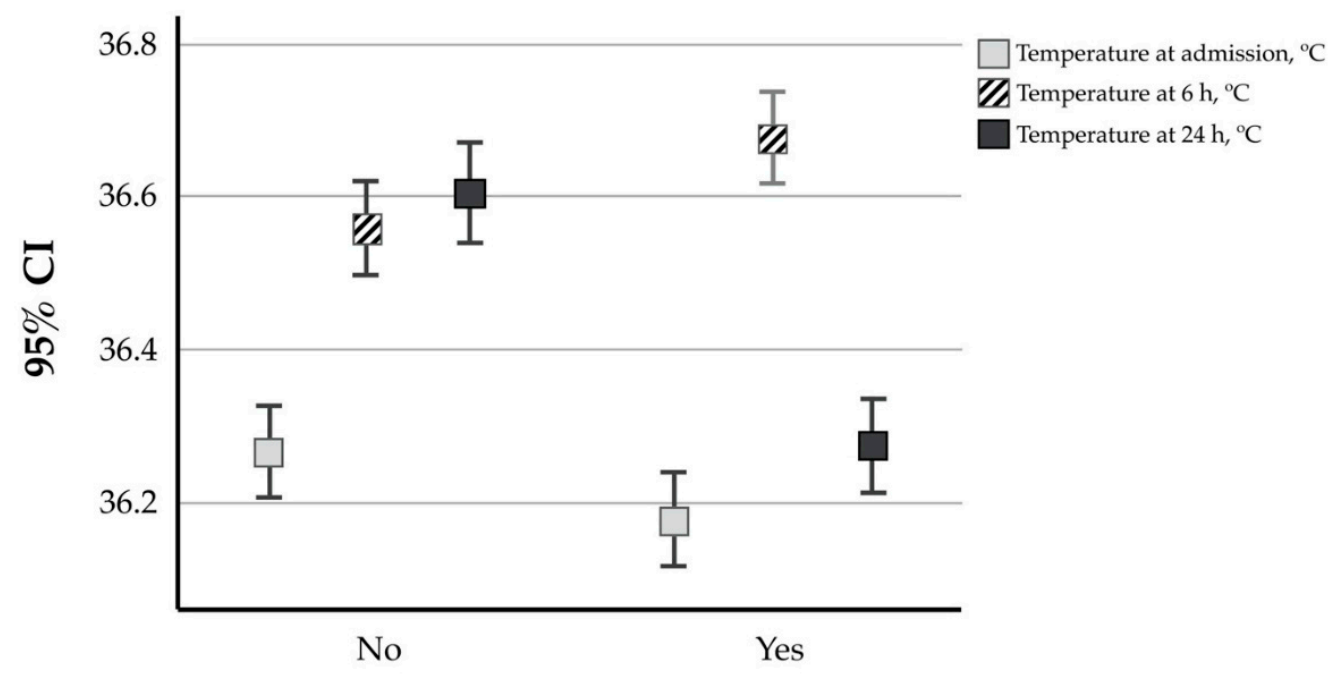

\section{Effective reperfusion}

Figure 1. Temperatures at admission and $6 \mathrm{~h} 24 \mathrm{~h}$ in relation to reperfusion effectiveness.

Pearson bivariate correlation analysis performed for the 3 different measurements showed a negative correlation between higher body temperature at admission $(r=-0.77, p=0.022)$ and at $24 \mathrm{~h}(r=-0.266 ; p<0.0001)$ with effective reperfusion, but no association was observed for the temperature at $6 \mathrm{~h}(r=0.055 ; p=0.103)$. Subsequently, we performed a logistic regression analysis, adjusted for variables that showed significant differences in the bivariate analysis (age, sex, onset-treatment time, blood glucose, atrial fibrillation, NIHSS at admission, leukoaraiosis, and HT) for effective reperfusion. Logistic regression analysis showed a non-significant association between the effectiveness of reperfusion therapy and higher body temperature at $6 \mathrm{~h}$ (OR: 1.178; 95\% CI: 0.934-1.484; $p=0.166$ ). Conversely, higher body temperature at admission and $24 \mathrm{~h}$ was negatively associated with effective reperfusion (OR: 0.700; 95\% CI: 0.552-0.887; $p=0.003$; OR: 0.373 and 95\% CI: 0.290-0.480; $p=0.001$ ) (Table 2).

Table 2. Logistic regression model for temperature at three different time points associated with effective reperfusion.

\begin{tabular}{cccc}
\hline & OR & CI 95\% & $p$ \\
\hline Temperature at admission & 0.700 & $0.552-0.887$ & 0.003 \\
Temperature at $6 \mathrm{~h}$ & 1.178 & $0.934-1.484$ & 0.166 \\
Temperature at $24 \mathrm{~h}$ & 0.373 & $0.290-0.480$ & 0.001 \\
\hline
\end{tabular}

* Adjusted for age, sex, onset-treatment time, blood glucose, atrial fibrillation, National Institute of Health Stroke Scale (NIHSS) score at admission, leukoaraiosis, and haemorrhagic transformation.

\subsection{Evaluation of Temperature and Functional Outcomes at 3 Months}

Among the 875 patients studied, 606 (69.3\%) showed good outcomes at 3 months compared to the remaining 269 (30.7\%) patients. Lower temperatures at admission and $24 \mathrm{~h}$ were associated with better outcomes at 3 months. Conversely, a higher body temperature at $6 \mathrm{~h}$ was associated with good outcomes at 3 months compared to those in the poor outcome group (Figure 2). 


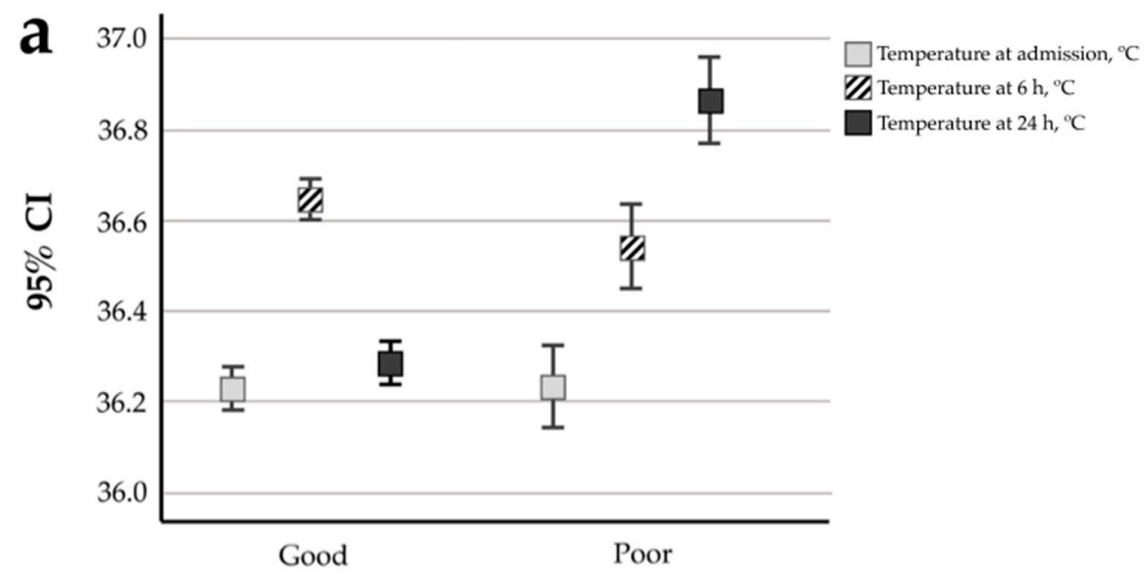

Outcome at 3 months

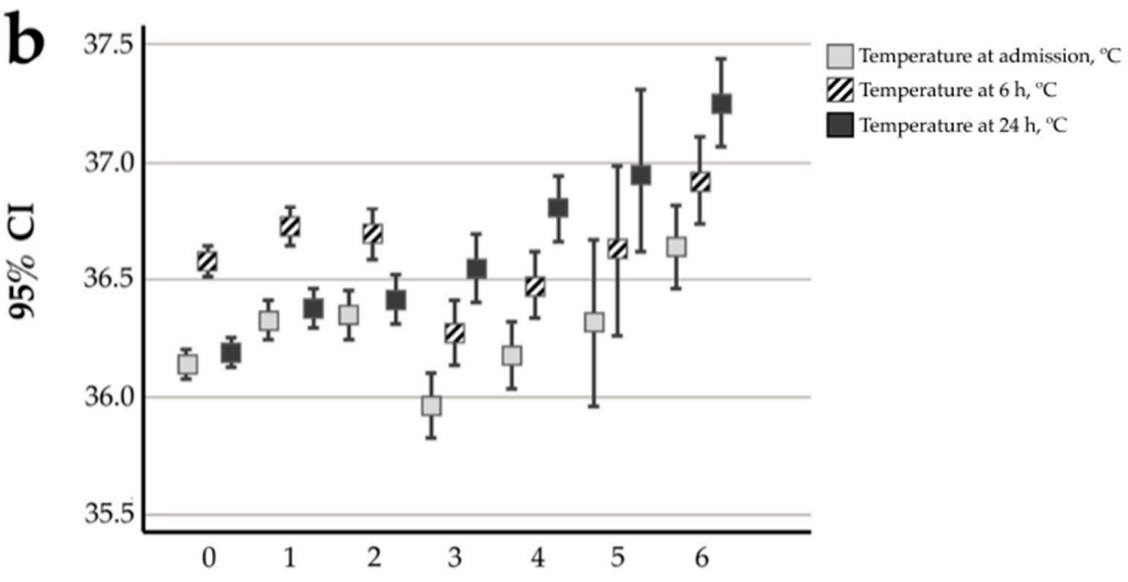

Rankin Scale at 3 months

Figure 2. Evaluation of functional outcomes at 3 months. (a) Temperatures at admission and 6 and $24 \mathrm{~h}$ in relation to patient outcomes. (b) Temperatures at admission and 6 and $24 \mathrm{~h}$ in relation to modified Rankin Scale scores at 3 months.

Logistic regression models were used to evaluate poor outcome at 3 months in relation to the temperatures at the three timepoints and adjusted by the variables that showed clinical and statistical significance in the model. The analysis showed no association with basal body temperature (OR 0.817, 95\% CI 0.475-1.405, $p=0.948)$ and temperature at $6 \mathrm{~h}$ (OR 0.792, 95\% CI 0.465-1.350, $p=0.792$ ). However, the temperature at $24 \mathrm{~h}$ was significantly associated with poor patient outcomes at 3 months (OR 2.190, 95\% CI 1.264-3.793, $p=0.005$ ) (Table 3).

Table 3. Logistic regression model for temperature at three different time points showing the relationships to poor outcomes at 3 months.

\begin{tabular}{cccc}
\hline & OR & CI 95\% & $p$ \\
\hline Temperature at admission & 0.817 & $0.475-1.405$ & 0.465 \\
Temperature at $6 \mathrm{~h}$ & 0.792 & $0.934-1.484$ & 0.792 \\
Temperature at $24 \mathrm{~h}$ & 2.190 & $1.264-3.793$ & 0.005 \\
\hline
\end{tabular}

* Adjusted for age, sex, onset-treatment time, blood glucose, atrial fibrillation, National Institute of Health Stroke Scale (NIHSS) score at admission, diffusion-weighted imaging (DWI) volume at admission, leukoaraiosis, and effective reperfusion. 
3.4. Determination of Temperature-Induced Biomarkers of Thrombolytic and Inflammatory Activity and Their Associations with Effective Reperfusion

cFN and MMP-9 serum levels showed a stronger correlation with temperature at $6 \mathrm{~h}(r=0.701$; $p<0.0001 ; r=0.697 ; p<0.0001)$ compared to IL-6 and TNF- $\alpha(r=0.113 ; p=0.001 ; r=0.223 ; p<0.0001)$. Similarly, a greater association was observed between IL- 6 and TNF- $\alpha$ levels and temperature at $24 \mathrm{~h}$ $(r=0.712 ; p<0.0001 ; r=0.602 ; p<0.0001)$ compared to those for cFN and MMP-9 $(r=0.195 ; p<0.0001$; $r=0.342 ; p<0.0001)$. Based on these findings, we performed multivariate analysis for each biomarker according to their most prominent correlation in relation to effective reperfusion. The analysis showed that MMP-9 levels at $6 \mathrm{~h}$ and IL- 6 at $24 \mathrm{~h}$ were independently associated with the effectiveness of the reperfusion therapy (OR: 1.004; 95\% CI: 1.002-1.006; $p<0.0001$ and OR: 0.979; 95\% CI: 0.967-0.992; $p=0.001)$. Table 4 shows the measured levels of all the studied biomarkers.

Table 4. Levers of biomarkers analysed at 6 and $24 \mathrm{~h}$.

\begin{tabular}{ccccc}
\hline & $N$ & Minimum & Maximum & Mean \pm Standard Deviation \\
\hline MMP-9 (6 h; ng/mL) & 799 & 3.6 & 430.1 & $103.81 \pm 79.16$ \\
Cellular fibronectin (6 h; ng/mL) & 804 & 4.1 & 35.9 & $8.55 \pm 4.55$ \\
TNF- $\alpha$ (Basal; ng/mL) & 831 & 1.8 & 45.8 & $16.84 \pm 6.65$ \\
IL-6 (Basal; ng/mL) & 841 & 1.1 & 58.6 & $11.17 \pm 10.00$ \\
MMP-9 (24 h; ng/mL) & 653 & 3.6 & 339.1 & $87.66 \pm 60.55$ \\
Cellular fibronectin $(24 \mathrm{~h} ; \mathrm{ng} / \mathrm{mL})$ & 662 & 1.7 & 18.3 & $7.11 \pm 2.260$ \\
TNF- $\alpha(24 \mathrm{~h} ; \mathrm{ng} / \mathrm{mL})$ & 669 & 7.3 & 65.8 & $27.98 \pm 9.16$ \\
IL-6 $(24 \mathrm{~h} ; \mathrm{ng} / \mathrm{mL})$ & 666 & 8.4 & 83.6 & $32.45 \pm 13.88$ \\
\hline
\end{tabular}

MMP-9: matrix metalloproteinase 9; TNF- $\alpha$ : tumour necrosis factor-alpha; IL-6: interleukin 6.

Simultaneous analysis of these two values in the logistic regression model revealed a positive association for MMP-9 levels at $6 \mathrm{~h}$ with effective reperfusion (OR: 1.008; 95\% CI: 1.005-1.010; $p<0.0001$ ). In contrast, IL- 6 at $24 \mathrm{~h}$ was an independent factor strongly associated with reperfusion failure (OR: 0.958; 95\% CI: 0.942-0.973; $p<0.0001$ ) (Table 5).

Table 5. Logistic regression model for the levels of biomarkers related to effective reperfusion.

\begin{tabular}{cccc}
\hline & OR * & 95\%CI & $p$ \\
\hline MMP-9 levels at 6 h $(\mathrm{ng} / \mathrm{mL})$ & 1.008 & $1.005-1.010$ & $<0.0001$ \\
IL-6 levels at 24 $\mathrm{h}(\mathrm{pg} / \mathrm{mL})$ & 0.958 & $0.942-0.973$ & $<0.0001$
\end{tabular}

* Adjusted for age, sex, onset-treatment time, blood glucose, atrial fibrillation, National Institute of Health Stroke Scale (NIHSS) score at admission, and leukoaraiosis. MMP-9: matrix metalloproteinase 9; IL-6: interleukin 6.

\subsection{Association of Temperature with HT and Infarct Volume}

In this study, $68 \%(n=595)$ of patients did not have HT. Among the patients who developed HT, IH1 was the most common subtype $(n=187,21.4 \%)$, followed by IH2 $(n=46,5.3 \%)$, PH1 $(n=26,3.0 \%)$, and PH2 $(n=21,2.4 \%)$. In all groups, temperature was directly related to haemorrhagic transformation and higher temperatures were observed in the most severe cases. Patients with no HT or IH showed an increase in temperature from admission to $6 \mathrm{~h}$ that then declined at $24 \mathrm{~h}$, while $\mathrm{PH}$ patients showed an increase in temperature over time. The multivariate analysis showed an association between $\mathrm{PH}$ and temperature (OR: 3.468; 95\% CI: 1.368-8.792, $p=0.009$ ), at 6 h (OR: 3.231; 95\% CI: 1.254-8.326; $p=0.015)$ and at $24 \mathrm{~h}$ (OR: $4.588 ; 95 \%$ CI: $1.726-12.199, p=0.002$ ).

Regarding infarct size, the mean infarct volume at days $4-7$ was $51.1 \pm 75.8 \mathrm{~mL}$. We observed no correlation between infarct volume and temperature at $6 \mathrm{~h}(r=0.066 ; p=0.066)$, although a significant correlation was observed at admission $(r=0.110 ; p=0.001)$ and at $24 \mathrm{~h}(r=0.302 ; p<0.0001)$. Multivariate analysis showed no association between infarct volume and temperature at admission (B: 6.956; 95\% CI: 0.620-14.531; $p=0.072$ ) or at 6 h (B: 6.765; 95\% CI: $0.725-14.253 ; p=0.077$ ). However, 
patients with higher temperatures at $24 \mathrm{~h}$ were over 20 times more likely to present greater infarct volumes between days 4 and 7 (B: 23.369; 95\% CI: 16.308-30.430; $p<0.0001$ ).

\section{Discussion}

Consistent with the findings of our previous study [2], higher body temperature at $24 \mathrm{~h}$ in the present study was negatively associated with effective reperfusion. This effect was also reflected by increased infarct volumes and poor outcome at 3 months in patients treated with rtPA. Higher body temperature at admission was also negatively associated with effective reperfusion but did not impair functional outcome at 3 months. Contrary to the observations at $24 \mathrm{~h}$, our analysis showed that temperature at $6 \mathrm{~h}$ in rtPA patients was not associated with poor outcome or infarct volume. Higher body temperature at $6 \mathrm{~h}$ was positively associated with effective reperfusion, although this association disappeared after adjusting for confounding factors in the logistic regression analysis.

Although the positive association observed between mild temperature at $6 \mathrm{~h}$ and effective reperfusion was weak, previous studies support the beneficial effect of mild hyperthermia in rtPA-treated patients [16,32-35]. Indeed, the results of in vitro studies on rtPA activity are consistent with this rationale and demonstrated a direct relationship between high body temperature and clot lysis effectiveness [20]. In this regard, in the Paracetamol (Acetaminophen) In Stroke (PAIS) trial aimed to assess whether early treatment (12-24 h) with paracetamol improved functional outcome in patients with acute stroke by reducing body temperature and preventing fever, reporting that this analgesic compound did not improve functional outcomes [32]. A second sub-analysis, derived from the same PAIS trial and designed to evaluate the influence of baseline body temperature on the effect of rtPA (alteplase) and functional outcome in patients with acute IS, found that high body temperature might have a larger benefit for treatment with alteplase than that in patients with lower body temperature [33]. In brief, during the initial $6 \mathrm{~h}$ window after cerebral infarction when rtPA treatment was provided, the results of our analysis suggested that the effect of high body temperature on clot lysis was more relevant in terms of functional prognosis than the use of neuroprotective strategies related to hypothermia. Therefore, efforts are required to develop novel approaches to improve thrombolytic therapy [36].

Analysis of thrombolytic biomarkers showed that MMP-9 levels at $6 \mathrm{~h}$ were directly correlated with temperature and with the effectiveness of reperfusion therapy, which could be explained by the increase in rtPA activity, although with an increased risk of developing HT, as was observed in the analysis. These findings suggest that the threshold of the beneficial effect of body temperature is very narrow. Indeed, the beneficial effect of body temperature at $6 \mathrm{~h}$ was observed at an average temperature of $36.6 \pm 0.7^{\circ} \mathrm{C}$.

As an inflammatory biomarker, IL-6 levels at $24 \mathrm{~h}$ were correlated with higher body temperature and negatively associated with effective reperfusion. High serum levels of inflammatory markers in the first $24 \mathrm{~h}$ were previously linked with early clinical deterioration and worsened outcome in acute IS [37-39]. These findings are further supported by our recent publication, in which IL-6 levels at $24 \mathrm{~h}$ were associated with worsened clinical outcomes in IS patients who underwent recanalisation therapy, regardless of the reperfusion effectiveness [40]. Therefore, delayed ( $24 \mathrm{~h})$ episodes of fever or high temperature may amplify the ischaemic lesion mediated by an inflammatory response, overcoming the benefit of rtPA treatment.

Based on these clinical findings, exhaustive monitoring of mild hyperthermia in clearly defined ranges during rtPA administration (in the first $6 \mathrm{~h}$ ) could have a beneficial effect on reperfusion therapies when combined with hypothermia strategies in later stages of the acute phase (i.e., at $24 \mathrm{~h}$ ).

The present study has some limitations. This single-centre study was conducted on a retrospectively enrolled patient registry. Transcranial Doppler used to determine the reperfusion rate could only be performed in $18 \%$ of the treated patients. Therefore, an 8-point or more improvement in NIHSS in the first $24 \mathrm{~h}$ was chosen as the endpoint as it was sensitive to the effects of early reperfusion, consistent with the results of other analyses [41,42], although the use of this clinical criterion to define effective 
reperfusion may lead to a bias in the classification of therapeutic response groups. The lack of imaging analysis to correlate the location of occlusions with the reperfusion effectiveness is also a critical issue that could interfere with our analysis, as patients with distal occlusions usually have higher reperfusion rates, small infarct sizes, and possibly low temperatures on admission (low inflammatory response). To overcome this critical limitation, in this study, we defined effective reperfusion as a reduction of $\geq 8$ points in the NIHSS in the first $24 \mathrm{~h}$, which normally involves patients with more proximal occlusions. Finally, as none of the patients included in this study reached hyperthermic values, we cannot rule out the possibility that temperatures higher than $37.5^{\circ} \mathrm{C}$ at $6 \mathrm{~h}$ could further promote effective reperfusion, although our data seems to predict such an effect.

In conclusion, the results of our analysis support the theory that mild hyperthermia could benefit reperfusion therapies by promoting clot lysis during the first $6 \mathrm{~h}$ of ischaemic stroke but worsen the outcome at later stages. Thrombolytic activity at $6 \mathrm{~h}$ seems to be related to temperature and may improve reperfusion therapies, warranting future studies to further elucidate the therapeutic potential of controlled mild hyperthermia in combination with rtPA administration.

Author Contributions: Conceptualization, P.Á.-G., J.C. and F.C.; Data curation, P.H., J.C. and T.S.; Formal analysis, P.Á.-G. and J.C.; Funding acquisition, J.C., T.S. and R.I.-R.; Investigation, M.R.-Y., I.L.-D. and J.M.P.; Methodology, M.R.-Y., J.M.P. and J.C.; Project administration, J.C., R.I.-R. and F.C.; Resources, A.D.S.-C., M.P.-M. and I.L.-D.; Software, A.D.S.-C., M.P.-M., I.L.-D. and F.C.; Supervision, J.C., T.S. and R.I.-R.; Validation, P.H., A.D.-C. and M.P.-M.; Visualization, P.Á.-G. and P.H.; Writing—original draft, P.Á.-G. and P.H.; Writing—review \& editing, J.C., T.S., R.I.-R. and F.C. All authors have read and agreed to the published version of the manuscript.

Funding: This study was partially supported by grants from the Spanish Ministry of Science and Innovation (SAF2017-84267-R), Xunta de Galicia (Consellería Educación: IN607A2018/3), Instituto de Salud Carlos III (ISCIII) (PI17/00540 and PI17/01103), Spanish Research Network on Cerebrovascular Diseases RETICS-INVICTUS PLUS (RD16/0019), and by the European Union FEDER program. Furthermore, Tomás. Sobrino (CPII17/00027) and Francisco Campos (CPII19/00020) are recipients of research contracts from the Miguel Servet Program of Instituto de Salud Carlos III. María Pérez-Mato is a Sara Borrell Researcher (CD19/00033). The sponsors did not participate in the study design, collection, analysis, or interpretation of the data, writing the report, or in the decision to submit the paper for publication.

Conflicts of Interest: The authors declare no conflicts of interest.

\section{References}

1. Castillo, J.; Davalos, A.; Marrugat, J.; Noya, M. Timing for fever-related brain damage in acute ischemic stroke. Stroke 1998, 29, 2455-2460. [CrossRef] [PubMed]

2. Millan, M.; Grau, L.; Castellanos, M.; Rodriguez-Yanez, M.; Arenillas, J.F.; Nombela, F.; Perez de la Ossa, N.; Lopez-Manzanares, L.; Serena, J.; Castillo, J.; et al. Body temperature and response to thrombolytic therapy in acute ischaemic stroke. Eur. J. Neurol. 2008, 15, 1384-1389. [CrossRef] [PubMed]

3. Saini, M.; Saqqur, M.; Kamruzzaman, A.; Lees, K.R.; Shuaib, A.; Investigators, V. Effect of hyperthermia on prognosis after acute ischemic stroke. Stroke 2009, 40, 3051-3059. [CrossRef] [PubMed]

4. Blanco, M.; Campos, F.; Rodriguez-Yanez, M.; Arias, S.; Fernandez-Ferro, J.; Gomez-Sanchez, J.C.; Castillo, J. Neuroprotection or increased brain damage mediated by temperature in stroke is time dependent. PLoS ONE 2012, 7, e30700. [CrossRef]

5. Dehkharghani, S.; Bowen, M.; Haussen, D.C.; Gleason, T.; Prater, A.; Cai, Q.; Kang, J.; Nogueira, R.G. Body Temperature Modulates Infarction Growth following Endovascular Reperfusion. AJNR Am. J. Neuroradiol. 2017, 38, 46-51. [CrossRef]

6. Forlivesi, S.; Micheletti, N.; Tomelleri, G.; Bovi, P.; Cappellari, M. Association of hyperglycemia, systolic and diastolic hypertension, and hyperthermia relative to baseline in the acute phase of stroke with poor outcome after intravenous thrombolysis. Blood Coagul. Fibrinolysis 2018, 29, 167-171. [CrossRef]

7. Greer, D.M.; Funk, S.E.; Reaven, N.L.; Ouzounelli, M.; Uman, G.C. Impact of fever on outcome in patients with stroke and neurologic injury: A comprehensive meta-analysis. Stroke 2008, 39, 3029-3035. [CrossRef]

8. De Jonge, J.C.; Wallet, J.; van der Worp, H.B. Fever worsens outcomes in animal models of ischaemic stroke: A systematic review and meta-analysis. Eur. Stroke J. 2019, 4, 29-38. [CrossRef] 
9. Wang, H.; Wang, B.; Normoyle, K.P.; Jackson, K.; Spitler, K.; Sharrock, M.F.; Miller, C.M.; Best, C.; Llano, D.; $\mathrm{Du}, \mathrm{R}$. Brain temperature and its fundamental properties: A review for clinical neuroscientists. Front. Neurosci. 2014, 8, 307. [CrossRef]

10. Basto, F.M.; Lyden, P. Hypothermia in acute ischemic stroke therapy. Handb. Clin. Neurol. 2018, 157, 823-837. [CrossRef]

11. Van der Worp, H.B.; Macleod, M.R.; Bath, P.M.; Demotes, J.; Durand-Zaleski, I.; Gebhardt, B.; Gluud, C.; Kollmar, R.; Krieger, D.W.; Lees, K.R.; et al. EuroHYP-1: European multicenter, randomized, phase III clinical trial of therapeutic hypothermia plus best medical treatment vs. best medical treatment alone for acute ischemic stroke. Int. J. Stroke 2014, 9, 642-645. [CrossRef] [PubMed]

12. Vieites-Prado, A.; Iglesias-Rey, R.; Fernandez-Susavila, H.; da Silva-Candal, A.; Rodriguez-Castro, E.; Grohn, O.H.; Wellmann, S.; Sobrino, T.; Castillo, J.; Campos, F. Protective Effects and Magnetic Resonance Imaging Temperature Mapping of Systemic and Focal Hypothermia in Cerebral Ischemia. Stroke 2016, 47, 2386-2396. [CrossRef]

13. Iglesias-Rey, R.; Rodriguez-Yanez, M.; Rodriguez-Castro, E.; Pumar, J.M.; Arias, S.; Santamaria, M.; Lopez-Dequidt, I.; Hervella, P.; Correa-Paz, C.; Sobrino, T.; et al. Worse Outcome in Stroke Patients Treated with rt-PA Without Early Reperfusion: Associated Factors. Transl. Stroke Res. 2018, 9, 347-355. [CrossRef] [PubMed]

14. Xiong, Y.; Manwani, B.; Fisher, M. Management of Acute Ischemic Stroke. Am. J. Med. 2019, 132, $286-291$. [CrossRef] [PubMed]

15. Kim, J.S. tPA Helpers in the Treatment of Acute Ischemic Stroke: Are They Ready for Clinical Use? J. Stroke 2019, 21, 160-174. [CrossRef]

16. Naess, H.; Idicula, T.; Lagallo, N.; Brogger, J.; Waje-Andreassen, U.; Thomassen, L. Inverse relationship of baseline body temperature and outcome between ischemic stroke patients treated and not treated with thrombolysis: The Bergen stroke study. Acta Neurol. Scand. 2010, 122, 414-417. [CrossRef] [PubMed]

17. Wrotek, S.E.; Kozak, W.E.; Hess, D.C.; Fagan, S.C. Treatment of fever after stroke: Conflicting evidence. Pharmacotherapy 2011, 31, 1085-1091. [CrossRef]

18. Khanevski, A.N.; Naess, H.; Thomassen, L.; Waje-Andreassen, U.; Nacu, A.; Kvistad, C.E. Elevated body temperature in ischemic stroke associated with neurological improvement. Acta Neurol. Scand. 2017, 136, 414-418. [CrossRef]

19. Mumme, A.; Kemen, M.; Homann, H.H.; Zumtobel, V. The temperature dependence of fibrinolysis with streptokinase. Dtsch. Med. Wochenschr. 1993, 118, 1594-1596. [CrossRef]

20. Yenari, M.A.; Palmer, J.T.; Bracci, P.M.; Steinberg, G.K. Thrombolysis with tissue plasminogen activator (tPA) is temperature dependent. Thromb. Res. 1995, 77, 475-481. [CrossRef]

21. Tsetis, D.K.; Katsamouris, A.N.; Giannoukas, A.D.; Hatzidakis, A.A.; Kostas, T.; Chamalakis, K.; Ioannou, C.; Gourtsoyiannis, N.C. Potential benefits from heating the high-dose rtPA boluses used in catheter-directed thrombolysis for acute/subacute lower limb ischemia. J. Endovasc. Ther. 2003, 10, 739-744. [CrossRef]

22. Mizuma, A.; Yenari, M.A. Anti-Inflammatory Targets for the Treatment of Reperfusion Injury in Stroke. Front. Neurol. 2017, 8, 467. [CrossRef] [PubMed]

23. Tsuji, K.; Aoki, T.; Tejima, E.; Arai, K.; Lee, S.R.; Atochin, D.N.; Huang, P.L.; Wang, X.; Montaner, J.; Lo, E.H. Tissue plasminogen activator promotes matrix metalloproteinase-9 upregulation after focal cerebral ischemia. Stroke 2005, 36, 1954-1959. [CrossRef] [PubMed]

24. Castellanos, M.; Leira, R.; Serena, J.; Pumar, J.M.; Lizasoain, I.; Castillo, J.; Davalos, A. Plasma metalloproteinase-9 concentration predicts hemorrhagic transformation in acute ischemic stroke. Stroke 2003, 34, 40-46. [CrossRef] [PubMed]

25. Meng, Q.; He, C.; Shuaib, A.; Wang, C.X. Hyperthermia worsens ischaemic brain injury through destruction of microvessels in an embolic model in rats. Int. J. Hyperth. 2012, 28, 24-32. [CrossRef] [PubMed]

26. Castellanos, M.; Leira, R.; Serena, J.; Blanco, M.; Pedraza, S.; Castillo, J.; Davalos, A. Plasma cellular-fibronectin concentration predicts hemorrhagic transformation after thrombolytic therapy in acute ischemic stroke. Stroke 2004, 35, 1671-1676. [CrossRef]

27. Leira, R.; Rodriguez-Yanez, M.; Castellanos, M.; Blanco, M.; Nombela, F.; Sobrino, T.; Lizasoain, I.; Davalos, A.; Castillo, J. Hyperthermia is a surrogate marker of inflammation-mediated cause of brain damage in acute ischaemic stroke. J. Intern. Med. 2006, 260, 343-349. [CrossRef] 
28. Rodriguez, J.A.; Sobrino, T.; Orbe, J.; Purroy, A.; Martinez-Vila, E.; Castillo, J.; Paramo, J.A. proMetalloproteinase-10 is associated with brain damage and clinical outcome in acute ischemic stroke. J. Thromb. Haemost. 2013, 11, 1464-1473. [CrossRef]

29. Alonso de Lecinana, M.; Egido, J.A.; Casado, I.; Ribo, M.; Davalos, A.; Masjuan, J.; Caniego, J.L.; Martinez Vila, E.; Diez Tejedor, E.; ad hoc Committee of the SEN Study Group for Cerebrovascular Diseases; et al. Guidelines for the treatment of acute ischaemic stroke. Neurologia 2014, 29, 102-122. [CrossRef]

30. Hacke, W.; Kaste, M.; Bluhmki, E.; Brozman, M.; Davalos, A.; Guidetti, D.; Larrue, V.; Lees, K.R.; Medeghri, Z.; Machnig, T.; et al. Thrombolysis with alteplase 3 to $4.5 \mathrm{~h}$ after acute ischemic stroke. N. Engl. J. Med. 2008, 359, 1317-1329. [CrossRef]

31. Sims, J.R.; Gharai, L.R.; Schaefer, P.W.; Vangel, M.; Rosenthal, E.S.; Lev, M.H.; Schwamm, L.H. ABC/2 for rapid clinical estimate of infarct, perfusion, and mismatch volumes. Neurology 2009, 72, 2104-2110. [CrossRef] [PubMed]

32. Den Hertog, H.M.; van der Worp, H.B.; van Gemert, H.M.; Algra, A.; Kappelle, L.J.; van Gijn, J.; Koudstaal, P.J.; Dippel, D.W.; Investigators, P. The Paracetamol (Acetaminophen) In Stroke (PAIS) trial: A multicentre, randomised, placebo-controlled, phase III trial. Lancet. Neurol. 2009, 8, 434-440. [CrossRef]

33. De Ridder, I.; den Hertog, H.; van Gemert, M.; Dippel, D.; van der Worp, B.; PAIS investigators. Increased benefit of alteplase in patients with ischemic stroke and a high body temperature. Cerebrovasc. Dis. 2013, 35, 60-63. [CrossRef] [PubMed]

34. Kim, S.H.; Saver, J.L. Initial body temperature in ischemic stroke: Nonpotentiation of tissue-type plasminogen activator benefit and inverse association with severity. Stroke 2015, 46, 132-136. [CrossRef] [PubMed]

35. Meunier, J.M.; Chang, W.T.; Bluett, B.; Wenker, E.; Lindsell, C.J.; Shaw, G.J. Temperature affects thrombolytic efficacy using rt-PA and eptifibatide, an in vitro study. Ther. Hypothermia Temp. Manag. 2012, 2, 112-118. [CrossRef]

36. Bonnard, T.; Gauberti, M.; Martinez de Lizarrondo, S.; Campos, F.; Vivien, D. Recent Advances in Nanomedicine for Ischemic and Hemorrhagic Stroke. Stroke 2019, 50, 1318-1324. [CrossRef]

37. Vila, N.; Castillo, J.; Davalos, A.; Chamorro, A. Proinflammatory cytokines and early neurological worsening in ischemic stroke. Stroke 2000, 31, 2325-2329. [CrossRef]

38. Bustamante, A.; Sobrino, T.; Giralt, D.; Garcia-Berrocoso, T.; Llombart, V.; Ugarriza, I.; Espadaler, M.; Rodriguez, N.; Sudlow, C.; Castellanos, M.; et al. Prognostic value of blood interleukin-6 in the prediction of functional outcome after stroke: A systematic review and meta-analysis. J. Neuroimmunol. 2014, 274, 215-224. [CrossRef]

39. Vila, N.; Castillo, J.; Davalos, A.; Esteve, A.; Planas, A.M.; Chamorro, A. Levels of anti-inflammatory cytokines and neurological worsening in acute ischemic stroke. Stroke 2003, 34, 671-675. [CrossRef]

40. Hervella, P.; Rodriguez-Castro, E.; Rodriguez-Yanez, M.; Arias, S.; Santamaria-Cadavid, M.; Lopez-Dequidt, I.; Estany-Gestal, A.; Maqueda, E.; Lopez-Loureiro, I.; Sobrino, T.; et al. Intra- and extra-hospital improvement in ischemic stroke patients: Influence of reperfusion therapy and molecular mechanisms. Sci. Rep. 2020, 10, 3513. [CrossRef]

41. Kharitonova, T.; Mikulik, R.O.; Roine, R.; Soinne, L.; Ahmed, N.; Wahlgren, N. Safe Implementation of Thrombolysis in Stroke Investigators.Association of Early National Institutes of Health Stroke Scale Improvement With Vessel Recanalization and Functional Outcome After Intravenous Thrombolysis in Ischemic Stroke. Stroke 2011, 42, 1638. [CrossRef] [PubMed]

42. Lansberg, G.M.; Christensen, S.; Kemp, S.; Mlynash, M.; Mishra, N.; Federau, C.; Tsai, P.J.; Kim, S.; Nogueria, G.R.; Jovin, T.; et al. Computed Tomographic Perfusion to Predict Response to Recanalization in Ischemic Stroke. Ann. Neurol. 2017, 81, 849. [CrossRef] [PubMed]

(C) 2020 by the authors. Licensee MDPI, Basel, Switzerland. This article is an open access article distributed under the terms and conditions of the Creative Commons Attribution (CC BY) license (http://creativecommons.org/licenses/by/4.0/). 